\section{Planejamento suicida entre adolescentes escolares: prevalência e fatores associados}

\author{
Suicide planning among teenage students: \\ prevalence and associated factors
}

\footnotetext{
1 Programa de Pós-graduação em Saúde Coletiva,

Universidade Luterana do

Brasil, Canoas, Brasil.

Correspondência L. S. Palazzo

Programa de Pós-graduação em Saúde Coletiva,

Universidade Luterana do Brasil.

Rua Duque de Caxias 888 , apto. 1006, Porto Alegre, RS 90010-280, Brasil.

li_palazzo@yahoo.com.br
}

\section{Abstract}

This cross-sectional study evaluated the prevalence of suicide planning and associated factors in a representative sample of 12- to-18-yearold students in Porto Alegre, Rio Grande do Sul State, Brazil. Two self-administered questionnaires were used to collect data: a questionnaire, standardized by the World Health Organization, about use of alcohol and drugs, violence, feelings of loneliness and sadness, family relationships, suicide planning, behavior in school, and relationships with friends; and the Body Shape Questionnaire. Prevalence of suicide planning was 6.3\%. Multivariate analysis using Cox regression showed that the prevalence of suicide planning was higher among girls and among adolescents that reported problems in the relationships with their parents. Drug use by friends and having a small number of close friends increased suicide planning by $90 \%$ and $66 \%$, respectively. Prevalence of suicide planning was two to three times greater among adolescents bullied by classmates or who reported feelings of loneliness or sadness. Adverse family relationships, aggressive interactions with classmates, and depressive symptoms increase the prevalence of suicide planning. Therefore, preventive measures should be developed in schools and should include the family.

Suicide; Adolescent; Family Conflict; Family Relations
Lissandra Baggio 1

Lílian S. Palazzo 1

Denise Rangel Ganzo de Castro Aerts 1

\section{Introdução}

O suicídio constitui-se num importante problema de saúde pública 1. Está entre as dez principais causas de morte na população mundial em todas as faixas etárias, ocupando o terceiro lugar no grupo com idade entre 15 e 34 anos 1 . As taxas variam em função do contexto social, gênero, meios utilizados e faixa etária 1,2,3. Entre adolescentes, os comportamentos de risco, em interação com fatores sociais e ambientais, têm gerado um aumento de mortes prematuras 1,3 .

Estudo realizado com dados de noventa países estimou em 7,4/100 mil a taxa de suicídios entre jovens de 15 e 19 anos. Entre aqueles com maiores taxas estão: Sri Lanka (46,5/100 mil), Lituânia (23,9/100 mil) e Rússia (23,6/100 mil). O Canadá aparece na 15a posição $(10,8 / 100 \mathrm{mil})$ e os Estados Unidos na 34a a (8,0/100 mil). No Brasil, a estimativa foi de 4,2/100 mil. Foram constatadas, também, taxas mais elevadas entre meninos $(10,5 / 100 \mathrm{mil})$ do que entre meninas $(4,1 / 100$ mil) ${ }^{4}$. Em outro estudo realizado com a população brasileira, entre 1980 e 2000, os autores verificaram um aumento de $32,8 \%$ na taxa masculina de suicídio, com crescimento em todos os grupos etários; entre as mulheres, as taxas são mais altas para o planejamento e tentativa de suicídio 5 .

A análise da evolução temporal da mortalidade por suicídio no Brasil, entre 1980 e 1999, mostrou que os estados da Região Sul apresentaramse acima da média nacional 6 . Nesse período, no 
Rio Grande do Sul, as taxas passaram de 9/100 mil para 11/100 mil 7. Porto Alegre (Rio Grande do Sul) e Curitiba (Paraná) foram as capitais com os mais altos índices entre os jovens ${ }^{8}$, quando comparadas às outras nove capitais, sinalizando a necessidade de um maior aprofundamento do estudo desse problema.

O comportamento suicida costuma ser concebido como variando em um continuum que se inicia com idéias de suicídio, ou seja, pensamentos de acabar com a própria vida. Se o processo avança, surge o planejamento suicida, que é a etapa em que o sujeito estabelece quando, onde e como fará para levar avante a conduta de autodestruição. A partir daí, poderá ocorrer a tentativa de suicídio, resultando ou não em morte 9,10.

A escola tem papel estratégico para a promoção e proteção da saúde dos alunos, pois é o local onde são reproduzidos os padrões de comportamentos e relacionamentos 11 que podem por em risco a saúde dos jovens. Nesse sentido, acredita-se que se a escola possa ser um local privilegiado para a identificação precoce de situações problemáticas, já que aspectos relacionados ao meio familiar, grupo de amigos e escola são de extrema importância para a qualidade de vida do adolescente 12,13.

Destaca-se que o comportamento suicida ocorre, muitas vezes, como reflexo de conflitos internos, sentimentos de depressão e ansiedade que acompanham a profunda reorganização física, psíquica e social que ocorre na adolescência. Entretanto, pode ser difícil determinar a intencionalidade de atitudes autodestrutivas. Em função disso, estudos são feitos para mapear um conjunto de fatores de risco que, individualmente ou associados, contribuem para a ação, tais como: uso de álcool e drogas, dificuldades nas relações familiares, transtornos alimentares, baixa auto-estima, exposição à violência, sentimentos depressivos, dentre outros 6,14,15. Assim, o presente estudo se propôs a investigar o planejamento suicida e fatores associados em adolescentes escolares de um município de porte médio da Região Metropolitana de Porto Alegre.

\section{Material e métodos}

O delineamento utilizado foi de estudo transversal, tendo como população alvo 2.282 alunos matriculados na $7 \underline{a}$ série (atualmente denominado 8 o ano) da rede pública municipal de Ensino Fundamental em Gravataí.

Para o cálculo do tamanho da amostra, utilizou-se como parâmetro uma prevalência de $50 \%$ de planejamento suicida, erro máximo aceito de $\pm 3,0 \%$ e nível de significância de 0,05 , totalizando
728 sujeitos. Com o objetivo de minimizar o efeito da amostragem por conglomerado, uma vez que se considerou a turma sorteada como um cluster, foi utilizado um efeito de delineamento de 1,5. Além disso, visando compensar possíveis perdas, ampliou-se a amostra em mais $20 \%$, chegandose a 1.312 escolares. Como esse número representava, aproximadamente, a metade dos alunos de 7ạ série, optou-se por sortear um número de turmas que correspondesse à metade mais uma de todas as turmas de cada uma das 15 regiões administrativas do município. Com isso, no final do processo, foram selecionados 1.366 alunos. Em função das perdas ocorridas (14,3\%), a amostra final foi composta por 1.170 estudantes, superior à inicialmente calculada (1.092 alunos). Com esse tamanho de amostra, o estudo tem poder de $80 \%$ para detectar significância estatística em razões de prevalências com magnitude igual ou superior a 1,5 .

Os dados foram coletados por acadêmicos da área da saúde, previamente capacitados, com o auxílio de três instrumentos, auto-aplicáveis, respondidos em sala de aula.

O primeiro questionário foi elaborado pela Organização Mundial da Saúde (OMS) 16, o Global School-based Student Health Survey, desenvolvido para avaliar a saúde de escolares. Desse instrumento, foram retiradas as variáveis relacionadas ao comportamento na escola; qualidade do relacionamento com colegas e familiares; consumo excessivo de álcool; absenteísmo escolar; sentimentos de tristeza, solidão e dificuldade para dormir; e o desfecho, planejamento suicida nos últimos trinta dias. Optouse por esse desfecho, pois, na adolescência, os pensamentos de morte são muito freqüentes e precisam ser diferenciados quanto à gravidade $\mathrm{e}$ intencionalidade. Nesse sentido, o planejamento suicida se aproxima da tentativa, pois, em geral, de cada cinco pessoas que planejam três efetivamente tentam o suicídio 14 .

O segundo questionário, elaborado especificamente para o estudo, forneceu dados referentes ao sexo e à inserção sócio-econômica, baseada na proposta da Associação Brasileira de Empresas de Pesquisa (ABEP) 17.

O terceiro instrumento foi o Body Shape Questionnaire (BSQ), validado por Cordás \& Castillo 18 , utilizado para avaliar a preocupação com a imagem corporal. Segundo os autores Cooper et al. 19, o BSQ recebe uma pontuação que é classificada em quatro grupos: (1) não preocupados com a imagem corporal (<81 pontos); (2) levemente preocupados (81 a 110 pontos); (3) moderadamente preocupados (111 a 140 pontos); e (4) extremamente preocupados (> 140 pontos). Para o presente estudo, tais categorias foram rea- 
grupadas em não preocupados, levemente preocupados e preocupados.

Para as análises univariadas e multivariadas, foi utilizada a regressão de Cox, modificada para estudos transversais ${ }^{20}$, com o objetivo de verificar a associação dos fatores em estudo com o planejamento suicida. Para a análise multivariada, foi criado um modelo hierarquizado, composto por quatro etapas. Na primeira etapa, foram introduzidas as variáveis cor da pele auto-referida, sexo e situação sócio-econômica. Na segunda etapa, introduziram-se os fatores em estudo: reação da família se chegar bêbado, uso de álcool pelos pais, pais entendem problemas, pais agridem, e faltar aula sem permissão.

Na terceira etapa, foram introduzidas as variáveis: uso de drogas por amigos, número de amigos próximos, agressão por colegas, ocorrência de injúrias, participação em brigas, sentimento de discriminação, medo de ir à escola e bom tratamento recebido dos colegas. Na quarta etapa, os fatores que ingressaram foram: uso excessivo de álcool (beber/ficar embriagado); sentimento de solidão; sentimento de tristeza; dificuldade para dormir; e percepção da imagem corporal.

Participaram da etapa seguinte à introdução no modelo aquelas variáveis que apresentaram associação com o desfecho com nível de significância $\leq 0,10$. Uma vez incluídas, as variáveis permaneceram até a última etapa da regressão. Para o modelo final, consideraram-se somente as variáveis que mostraram nível de significância $<0,05$. As razões de prevalência, intervalos de confiança e níveis de significância apresentados referem-se aos valores encontrados na etapa em que a variável foi introduzida no modelo, com o ajuste das variáveis das hierarquias superiores e da mesma etapa.

Como etapa anterior à coleta dos dados, foram feitas reuniões com o corpo diretivo das escolas para esclarecimentos sobre o estudo e com os pais ou responsáveis pelos alunos, para a obtenção da assinatura do Termo de Consentimento Livre e Esclarecido. Este estudo é parte integrante de um projeto maior denominado A Saúde do Escolar da Rede Pública Municipal de Gravataí$R S$, aprovado pelo Comitê de Ética em Pesquisa da Universidade Luterana do Brasil (ULBRA; nº. 2004-375H)

\section{Resultados}

A distribuição dos estudantes, segundo as variáveis sócio-demográficas, revela que a amostra é formada, majoritariamente, por adolescentes que se autodefiniram como brancos $(52,6 \%)$; $59 \%$ integrando a categoria sócio-econômica intermediária (C); $52,5 \%$ pertencendo ao sexo feminino e a média de idade foi de 14 anos (desvio-padrão - $\mathrm{DP}=1,13$ ).

A prevalência encontrada de planejamento suicida, nos últimos trinta dias, foi de $6,3 \%$. Em relação aos fatores em estudo, verificou-se que cor da pele e inserção sócio-econômica não apresentaram associação estatisticamente significativa com o desfecho na análise multivariada. A variável sexo manteve a associação já apresentada na análise univariada, mostrando que os adolescentes do sexo masculino referiram $40 \%$ menos planejamento suicida do que os do sexo feminino (Tabela 1).

Em relação às variáveis introduzidas na segunda etapa da regressão (Tabela 1), apenas o uso de álcool pelos pais foi excluído do modelo, em função da ausência de associação; as demais se associaram ao desfecho. Cerca da metade $(51 \%)$ dos estudantes relatou que não sabia a reação da família se eles chegassem bêbados a casa e, para aqueles cuja expectativa era a indiferença familiar, a prevalência do desfecho foi $96 \%$ maior. Os jovens que se sentiram, às vezes, entendidos pelos pais relataram terem planejado suicídio cerca de duas vezes mais do que os que se sentiram sempre entendidos. Da mesma forma, aqueles que referiram ser agredidos pelos pais ou responsáveis e os que faltaram às aulas sem o conhecimento e a permissão deles apresentaram uma prevalência do desfecho 2,7 vezes maior do que seus pares de referência.

Na terceira etapa da regressão (Tabela 2), das oito variáveis que ingressaram no modelo, somente três se associaram ao desfecho: amigos usam drogas, número de amigos próximos e agressão por colegas. Os amigos usarem drogas e o jovem ter poucos amigos próximos aumentaram, respectivamente, em $90 \%$ e $66 \%$ a prevalência de planejamento suicida. Ainda maior é a freqüência de adolescentes que planejaram acabar com a própria vida entre aqueles que relataram terem sido agredidos pelos colegas. As variáveis ocorrência de injúrias, participação em brigas, sentimento de discriminação e medo de ir à escola por insegurança associaram-se ao desfecho apenas na análise univariada.

Por fim, na quarta etapa (Tabela 2), além das variáveis selecionadas anteriormente, foram incluídas no modelo as variáveis: beber/ficar embriagado, sentimento de solidão, dificuldade para dormir, sentimento de tristeza e imagem corporal. Nas análises univariadas, tais variáveis apresentaram associação estatisticamente significativa com o planejamento suicida. No entanto, na multivariada, somente os sentimentos de tristeza e de solidão mostraram-se associados ao desfecho. Os adolescentes que referiram senti- 
Resultados das análises univariadas e das primeira e segunda etapas da análise multivariada entre planejamento suicida e fatores em estudo. Gravataí, Rio Grande do Sul, Brasil, 2005.

\begin{tabular}{|c|c|c|c|c|c|c|}
\hline \multirow[t]{2}{*}{ Variáveis } & \multirow[t]{2}{*}{$\mathbf{n}$} & \multicolumn{3}{|c|}{ Univariada } & \multicolumn{2}{|c|}{ Multivariada } \\
\hline & & n (\%) & RP (IC95\%) & Valor de $p$ & RP (IC95\%) & Valor de $p$ \\
\hline \multicolumn{7}{|l|}{ 1ạ Etapa } \\
\hline \multicolumn{7}{|l|}{ Cor da pele } \\
\hline Brancos & 615 & $41(6,7)$ & 1,00 & - & 1,00 & - \\
\hline Não brancos & 555 & $33(5,9)$ & $0,89(0,57-1,39)$ & 0,608 & $0,91(0,58-1,42)$ & 0,668 \\
\hline \multicolumn{7}{|l|}{ Sexo } \\
\hline Feminino & 614 & $48(7,8)$ & 1,00 & - & 1,00 & - \\
\hline Masculino & 555 & $26(4,7)$ & $0,60(0,38-0,95)$ & 0,030 & $0,60(0,38-0,96)$ & 0,032 \\
\hline \multicolumn{7}{|c|}{ Situação sócio-econômica } \\
\hline Classe B & 256 & $12(4,7)$ & 1,00 & - & 1,00 & - \\
\hline Classe C & 688 & $49(7,1)$ & $1,52(0,82-2,81)$ & 0,181 & $1,49(0,81-2,76)$ & 0,200 \\
\hline Classe D e E & 226 & $13(5,7)$ & $1,23(0,57-2,63)$ & 0,600 & $1,18(0,55-2,56)$ & 0,665 \\
\hline \multicolumn{7}{|l|}{ 2a Etapa } \\
\hline \multicolumn{7}{|c|}{ Reação da família se chegar bêbado } \\
\hline Importa-se & 494 & $27(5,5)$ & 1,00 & & 1,00 & - \\
\hline Indiferente & 80 & $11(13,7)$ & $2,51(1,30-4,86)$ & 0,006 & $1,96(1,00-3,83)$ & 0,048 \\
\hline Desconhecida & 596 & $36(6,0)$ & $1,10(0,68-1,79)$ & 0,692 & $0,99(0,61-1,61)$ & 0,982 \\
\hline \multicolumn{7}{|c|}{ Uso de álcool pelos pais } \\
\hline Não & 387 & $17(4,4)$ & 1,00 & - & 1,00 & - \\
\hline Sim & 739 & $55(7,4)$ & $1,70(0,99-2,89)$ & 0,051 & $1,44(0,85-2,42)$ & 0,171 \\
\hline \multicolumn{7}{|c|}{ Pais entendem problemas/preocupações } \\
\hline Sim & 601 & $21(3,5)$ & 1,00 & - & 1,00 & - \\
\hline Às vezes & 309 & $32(10,4)$ & $2,96(1,74-5,05)$ & 0,000 & $2,25(1,30-3,89)$ & 0,004 \\
\hline Não & 260 & $21(8,1)$ & $2,32(1,29-4,17)$ & 0,005 & $1,68(0,91-3,10)$ & 0,098 \\
\hline \multicolumn{7}{|l|}{ Pais agridem } \\
\hline Não & 982 & $45(4,6)$ & 1,00 & - & 1,00 & - \\
\hline $\operatorname{Sim}$ & 188 & $29(15,4)$ & $3,36(2,16-5,22)$ & 0,000 & $2,72(1,73-4,30)$ & 0,000 \\
\hline \multicolumn{7}{|c|}{ Faltar aula sem permissão } \\
\hline Não & 934 & $48(5,1)$ & 1,00 & - & 1,00 & - \\
\hline Sim & 236 & $26(11,0)$ & $2,14(1,36-3,38)$ & 0,001 & $2,72(1,05-2,66)$ & 0,032 \\
\hline
\end{tabular}

RP: razão de prevalência; IC95\%: intervalo de 95\% de confiança.

rem-se sozinhos e os tristes apresentaram cerca de três vezes mais planejamento suicida do que os adolescentes que não referiram esses sentimentos.

O modelo final apresenta as variáveis que mostraram associação estatisticamente significativa com o planejamento suicida na análise multivariada (Tabela 3).

\section{Discussão}

O comportamento suicida em adolescentes vem sendo alvo de muitas pesquisas. Porém, relativamente poucas têm dado atenção específica aos fatores associados à etapa em que o sujeito não apenas pensa, mas já planeja como fará pa- ra acabar com a própria vida, sendo essa uma importante contribuição do presente estudo. Assim, utilizando-se uma amostra representativa de adolescentes escolares de um município da Região Metropolitana de Porto Alegre, encontrou-se uma prevalência $6,3 \%$ de planejamento suicida. Este resultado reveste-se de importância para a saúde coletiva, em função do caráter multiplicador dessa situação. Em estudo realizado com escolares britânicos, foi verificado que o comportamento de auto-injúria entre jovens, que pode preceder atos suicidas, está fortemente associado à presença de colegas que apresentaram esse tipo de atitude 21 .

Embora a literatura aponte para um aumento do comportamento suicida entre os adolescentes brancos 22 , neste estudo não se verificou diferen- 
Tabela 2

Resultados das análises univariadas e das terceira e quarta etapas da análise multivariada entre planejamento suicida e fatores em estudo. Gravataí, Rio Grande do Sul, Brasil, 2005.

\begin{tabular}{|c|c|c|c|c|c|c|}
\hline \multirow[t]{2}{*}{ Variáveis } & \multirow[t]{2}{*}{$\mathbf{n}$} & \multicolumn{3}{|c|}{ Univariada } & \multicolumn{2}{|c|}{ Multivariada } \\
\hline & & n (\%) & RP (IC95\%) & Valor de $p$ & RP (IC95\%) & Valor de $\mathrm{p}$ \\
\hline \multicolumn{7}{|l|}{ 3a Etapa } \\
\hline \multicolumn{7}{|l|}{ Amigos usam drogas } \\
\hline Não & 827 & $35(4,2)$ & 1,00 & - & 1,00 & - \\
\hline Sim & 343 & $39(11,4)$ & $2,68(1,73-4,16)$ & 0,000 & $1,90(1,21-2,99)$ & 0,005 \\
\hline \multicolumn{7}{|l|}{ Número de amigos próximos } \\
\hline$\geq 3$ & 986 & $56(5,7)$ & 1,00 & - & 1,00 & - \\
\hline$<3$ & 183 & $18(9,8)$ & $1,73(1,04-2,88)$ & 0,034 & $1,66(1,02-2,70)$ & 0,042 \\
\hline \multicolumn{7}{|l|}{ Agressão por colegas } \\
\hline Não & 1.097 & $64(5,8)$ & 1,00 & & 1,00 & \\
\hline Sim & 73 & $10(13,7)$ & $2,35(1,26-4,37)$ & 0,007 & $2,24(1,22-4,12)$ & 0,009 \\
\hline \multicolumn{7}{|l|}{ Ocorrência de injúrias } \\
\hline Não & 679 & $34(5,0)$ & 1,00 & - & 1,00 & - \\
\hline Acidentalmente & 396 & $28(7,1)$ & $1,41(0,87-2,29)$ & 0,165 & $1,37(0,84-2,25)$ & 0,210 \\
\hline Alguém me machucou & 77 & $10(13,0)$ & $2,59(1,33-5,03)$ & 0,005 & $1,22(0,57-2,63)$ & 0,603 \\
\hline Machuquei-me por querer & 18 & $2(11,1)$ & $2,22(0,58-8,53)$ & 0,247 & $1,12(0,25-4,92)$ & 0,881 \\
\hline \multicolumn{7}{|l|}{ Participação em brigas } \\
\hline Não & 947 & $46(4,9)$ & 1,00 & - & 1,00 & - \\
\hline Sim & 223 & $28(12,6)$ & $2,59(1,66-4,06)$ & 0,000 & $1,59(0,96-2,61)$ & 0,068 \\
\hline \multicolumn{7}{|l|}{ Sentimento de discriminação } \\
\hline Não & 924 & $46(5,0)$ & 1,00 & - & 1,00 & - \\
\hline Sim & 246 & $28(11,4)$ & $2,28(1,46-3,58)$ & 0,000 & $1,39(0,84-2,30)$ & 0,202 \\
\hline \multicolumn{7}{|l|}{ Medo de ir à escola } \\
\hline Não & 1.056 & $60(5,7)$ & 1,00 & - & 1,00 & - \\
\hline Sim & 114 & $14(12,2)$ & $2,16(1,25-3,74)$ & 0,006 & $1,40(0,80-2,45)$ & 0,232 \\
\hline \multicolumn{7}{|l|}{ Colegas tratam bem } \\
\hline Sim & 593 & $39(6,6)$ & 1,00 & - & 1,00 & \\
\hline Às vezes & 357 & $25(7,0)$ & $1,06(0,66-1,73)$ & 0,800 & $0,84(0,52-1,34)$ & 0,468 \\
\hline Não & 220 & $10(4,6)$ & $0,69(0,35-1,37)$ & 0,291 & $0,69(0,35-1,37)$ & 0,289 \\
\hline \multicolumn{7}{|l|}{ 4a Etapa } \\
\hline \multicolumn{7}{|l|}{ Beber/Ficar de porre } \\
\hline Não bebeu & 460 & $15(3,3)$ & 1,00 & - & 1,00 & - \\
\hline Bebeu mas não ficou de porre & 525 & $35(6,7)$ & $2,05(1,13-3,70)$ & 0,018 & $1,59(0,88-2,86)$ & 0,122 \\
\hline Sim, ficou de porre & 185 & $24(13,0)$ & $3,98(2,13-7,41)$ & 0,000 & $0,60(0,81-3,16)$ & 0,179 \\
\hline \multicolumn{7}{|l|}{ Sentimento de solidão } \\
\hline Não & 571 & $19(3,3)$ & 1,00 & - & 1,00 & - \\
\hline Às vezes & 518 & $33(6,4)$ & $1,91(1,10-3,32)$ & 0,021 & $1,40(0,77-2,51)$ & 0,265 \\
\hline Sim & 80 & $22(27,5)$ & $8,26(4,68-14,58)$ & 0,000 & $2,83(1,40-5,71)$ & 0,004 \\
\hline \multicolumn{7}{|l|}{ Dificuldade para dormir } \\
\hline Não & 710 & $29(4,1)$ & 1,00 & - & 1,00 & - \\
\hline Às vezes & 425 & $33(7,8)$ & $1,90(1,17-3,08)$ & 0,009 & $0,81(0,44-1,49)$ & 0,502 \\
\hline Sim & 34 & $12(35,3)$ & $8,64(4,85-15,41)$ & 0,000 & $1,42(0,60-3,35)$ & 0,419 \\
\hline \multicolumn{7}{|l|}{ Sentimento de tristeza } \\
\hline Não & 930 & $29(3,1)$ & 1,00 & - & 1,00 & - \\
\hline Sim & 240 & $45(18,8)$ & $6,04(3,87-9,42)$ & 0,000 & $3,17(1,84-5,47)$ & 0,000 \\
\hline \multicolumn{7}{|l|}{ Imagem corporal } \\
\hline Não preocupado & 890 & $44(4,9)$ & 1,00 & - & 1,00 & - \\
\hline Levemente preocupado & 169 & $17(10,1)$ & $2,03(1,19-3,47)$ & 0,009 & $0,98(0,52-1,83)$ & 0,941 \\
\hline Preocupado & 106 & $13(12,3)$ & $2,48(1,38-4,451)$ & 0,002 & $1,23(0,63-2,41)$ & 0,542 \\
\hline
\end{tabular}

RP: razão de prevalência; IC95\%: intervalo de 95\% de confiança. 


\begin{tabular}{|c|c|c|}
\hline Variáveis & RP (IC95\%) & $p$ \\
\hline \multicolumn{3}{|l|}{ Sexo } \\
\hline Feminino & 1,00 & - \\
\hline Masculino & $0,60(0,38-0,96)$ & 0,032 \\
\hline \multicolumn{3}{|c|}{ Reação da família se chegar bêbado } \\
\hline Importa-se & 1,00 & - \\
\hline Indiferente & $1,96(1,00-3,83)$ & 0,048 \\
\hline Desconhecida & $0,99(0,61-1,61)$ & 0,982 \\
\hline \multicolumn{3}{|c|}{ Pais entendem problemas/preocupações } \\
\hline Sim & 1,00 & - \\
\hline Às vezes & $2,25(1,30-3,89)$ & 0,004 \\
\hline Não & $1,68(0,91-3,10)$ & 0,098 \\
\hline \multicolumn{3}{|l|}{ Pais agridem } \\
\hline Não & 1,00 & - \\
\hline Sim & $2,72(1,73-4,30)$ & 0,000 \\
\hline \multicolumn{3}{|c|}{ Faltar aula sem permissão } \\
\hline Não & 1,00 & - \\
\hline Sim & $2,72(1,05-2,66)$ & 0,032 \\
\hline \multicolumn{3}{|c|}{ Amigos usam drogas } \\
\hline Não & 1,00 & - \\
\hline Sim & $1,90(1,21-2,99)$ & 0,005 \\
\hline \multicolumn{3}{|c|}{ Número de amigos próximos } \\
\hline$\geq 3$ & 1,00 & - \\
\hline$<3$ & $1,66(1,02-2,70)$ & 0,042 \\
\hline \multicolumn{3}{|c|}{ Agressão por colegas } \\
\hline Não & 1,00 & - \\
\hline Sim & $2,24(1,22-4,12)$ & 0,009 \\
\hline \multicolumn{3}{|c|}{ Sentimento de solidão } \\
\hline Não & 1,00 & - \\
\hline Às vezes & $1,40(0,77-2,51)$ & 0,265 \\
\hline Sim & $2,83(1,40-5,71)$ & 0,004 \\
\hline \multicolumn{3}{|c|}{ Sentimento de tristeza } \\
\hline Não & 1,00 & - \\
\hline Sim & $3,17(1,84-5,47)$ & 0,000 \\
\hline
\end{tabular}

RP: razão de prevalência; IC95\%: intervalo de 95\% de confiança.

ça significativa em relação à cor da pele e à inserção sócio-econômica, corroborando resultados encontrados por outros autores em pesquisa sobre o tema no Brasil 23. O resultado pode ter ocorrido em função de se ter estudado jovens de escolas públicas, bastante semelhantes em relação à sua inserção sócio-econômica e composição racial. Sabe-se que a vulnerabilidade racial/étnico está relacionada, habitualmente, a diferenças sociais, culturais e econômicas 24 , fato que não parece acontecer, de forma marcante, entre os jovens estudados. Botega \& Werlang 25 destacam que o risco maior é estar nos extremos, em estratos econômicos mais ricos ou mais pobres, o que não é o caso, visto que a maioria dos adolescen- tes encontrava-se na categoria de inserção sócioeconômica intermediária (categoria C).

A variável sexo associou-se ao desfecho, mostrando maior prevalência de planejamento suicida entre jovens do sexo feminino. Esse achado é corroborado por outros autores sobre o tema, que verificaram que as meninas planejam e tentam mais; no entanto são os meninos que efetivam o suicídio 23,26. Uma das possíveis explicações refere-se à maior prevalência de depressão 27 e tendência à introspecção entre as meninas, enquanto os meninos tendem mais à ação ${ }^{28}$. Esse achado vai ao encontro de Wermeiren et al. 29 que referem que a exposição à violência, os sentimentos depressivos e o risco de suicídio em ado- 
lescentes apresentam importantes diferenças entre os gêneros.

Em relação às variáveis familiares investigadas, revestem-se de importância os resultados encontrados, sobretudo pela possibilidade de realização de ações preventivas dentro da escola. Ser agredido pelos pais; a expectativa de indiferença parental diante de situação de risco vivida pelo jovem, como a embriaguez; a falta de limites, representada pelo absenteísmo sem permissão; e se sentir compreendido pelos pais apenas esporadicamente, refletem um contexto familiar de violência doméstica e dificuldades de comunicação. Essas situações, por si sós, são consideradas como de risco para o suicídio 26,30. Além disso, podem gerar sentimentos de abandono, descaso e insegurança. Esses sentimentos formam a base de quadros depressivos em que o suicídio é uma das principais conseqüências, especialmente entre jovens 21,26,30.

A relação entre depressão e suicídio também se vê refletida nesse estudo, pois os adolescentes que se sentiam mais sozinhos e/ou tristes apresentaram prevalências mais altas de planejamento suicida do que aqueles sem esse tipo de sentimentos.

Adolescentes com manifestações suicidas apresentam história positiva para situações de violência, brigas e agressões, transgressão às leis e problemas de conduta em geral 31 . No presente estudo, a análise univariada mostrou associação entre planejamento suicida e ter sofrido injúria intencional, participação em brigas, sentimento de discriminação e faltar à aula por insegurança. Todavia, na análise multivariada, essas associações perderam significância estatística, quando foram controladas pelas outras variáveis introduzidas nesta etapa. Na adolescência, a identificação com amigos e o sentimento de pertencer a um grupo são muito importantes na estruturação da personalidade, tendo efeito de apoio e proteção ${ }^{32}$. Contudo, adolescentes com manifestações suicidas têm relacionamentos pobres e tendem a fazer amizades com jovens também problemáticos $33 \mathrm{ou}$, ainda, podem reatualizar, no grupo de iguais, agressões sofridas na família e serem rechaçados. Tudo isso intensifica sentimentos de solidão e de que o mundo lhes é hostil e não continente, reforçando, não raro, o comportamento suicida 34 .

O consumo abusivo de álcool, dificuldade para dormir e preocupação com a imagem corporal mostraram-se associados ao desfecho somente na análise univariada, perdendo significância quando passaram a ser controlados pelas variáveis sentimento de solidão e tristeza. Como dito anteriormente, a presença de sintomas depressivos é um importante fator de risco para comportamento suicida, situando-se como um dos mais fortes preditores dessa condição 21,30,35,36. Esse dado é relevante, particularmente para a prevenção do problema, visto que a depressão pode passar despercebida por familiares, professores e profissionais da saúde, porque, na adolescência, esse transtorno muitas vezes se manifesta por intermédio de queixas somáticas, problemas no âmbito sexual, baixo rendimento escolar e problemas de conduta, em vez de humor deprimido 37.

O estudo mostrou que relações familiares adversas, pequeno número de amigos, contatos agressivos com colegas e sintomas depressivos aumentam a prevalência de planejamento suicida. Entretanto, esses resultados devem ser interpretados considerando-se as características do estudo que, por ter um delineamento transversal, não permite o estabelecimento de nexo causal entre as variáveis e o desfecho. Além disso, por utilizar uma amostra representativa de estudantes da rede escolar pública, os dados não podem ser estendidos para a totalidade dos adolescentes do município, já que os da rede privada e aqueles que estão fora do sistema de ensino poderiam apresentar resultados diferentes. Acredita-se que os dados obtidos, possivelmente, traduzem o que ocorre com jovens escolares da rede pública de comunidades semelhantes, especialmente no que diz respeito às condições sócio-econômicas. Assim, pode servir de base ao planejamento de ações preventivas com base na escola em parceria com outros setores da sociedade, como o setor saúde. Entre elas, destaca-se a efetiva capacitação dos professores a fim de que possam trabalhar temas importantes da vida desses jovens, identificar adolescentes em risco e realizar ações que propiciem o maior envolvimento da família. 


\section{Resumo}

Estudo transversal com objetivo de investigar a prevalência de planejamento suicida e fatores associados em amostra representativa de adolescentes escolares de um município da grande Porto Alegre, Rio Grande do Sul, Brasil, com idade entre 12 e 18 anos. Para a coleta de dados, utilizaram-se dois questionários auto-aplicáveis: um sobre uso de álcool, drogas, violência, sentimentos de solidão e tristeza, relacionamento familiar, planejamento suicida, comportamento na escola e com amigos, padronizado pela Organização Mundial da Saúde, e o Body Shape Questionnaire. A prevalência de planejamento suicida foi 6,3\%. A análise multivariada utilizando a regressão de Cox mostrou que o planejamento suicida é mais prevalente em meninas e jovens que referem problemas na relação com os pais. $O$ uso de drogas pelos amigos e pequeno número de amigos próximos aumentaram em, respectivamente, $90 \%$ e $66 \%$ o planejamento suicida. Aqueles agredidos por colegas, os que referiram sentirem-se sozinhos e tristes apresentaram duas a três vezes mais prevalência de planejamento suicida. Assim, relações familiares adversas, contatos agressivos com colegas $e$ sintomas depressivos aumentam a prevalência de planejamento suicida, necessitando de ações preventivas na escola, incluindo a família.

Suicídio; Adolescente; Conflito Familiar; Relações Familiares

\section{Colaboradores}

L. Baggio participou da redação do artigo. L. S. Palazzo colaborou na redação e revisão do artigo. D. R. G. C. Aerts coordenou o trabalho de campo, realizou a análise dos dados e a revisão do artigo.

\section{Referências}

1. World Health Organization. The world health report 2001. http://www.who.int/whr/2001/charper2/en/index6.html (acessado em 05/Abr/2008).

2. Berghammer R, Hajnal A. Suicide in children and adolescents. Acta Psychiatr Scand 2004; 110:315.

3. Evans E, Hawton K, Rodham K, Deeks J. The prevalence of suicidal phenomena in adolescents: a systematic review of population-based studies. Suicide Life Threat Behav 2005; 35:239-50.

4. Wasserman D, Cheng Q, Jiang GX. Global suicide rates among young people aged 15-19. World Psychiatry 2005; 4:114-20.

5. Barros MBA, Oliveira HB, Marín-León L. Epidemiologia no Brasil. In: Werlang BG, Botega NJ, organizadores. Comportamento suicida. Porto Alegre: Editora ArtMed; 2004. p. 45-58.

6. Souza ER, Minayo MCS. Análise temporal da mortalidade por causas externas no Brasil: décadas de 80 e 90. In: Minayo MCS, Souza ER, organizadores. Violência sob o olhar da saúde infrapolítica. Rio de Janeiro: Editora Fiocruz; 2003. p. 83-107.
7. Meneghel SN, Victora CG, Faria NMX, Carvalho LA, Falk JW. Características epidemiológicas do suicídio no Rio Grande do Sul. Rev Saúde Pública 2004; 38:804-10.

8. Souza ER, Minayo MCS, Malaquias JV. Suicide among young people in selected Brazilian State capitals. Cad Saúde Pública 2002; 18:673-83.

9. Peña Galván LY, Casas Rodríguez L, Padilla de la Cruz M, Gómez Arencibia T, Gallardo Alvarez M. Comportamiento del intento suicida en un grupo de adolescentes y jóvenes. Rev Cuba Med Mil 2002; 31:182-7.

10. Serfaty E. Suicidio en la adolescencia. Adolesc Latinoam 1998; 1:105-10.

11. Haavet OR, Dalen I, Straand J. Depressive symptoms in adolescent pupils are heavily influenced by the school they go to. A study of 10th grade pupils in Oslo, Norway. Eur J Public Health 2006; 16:400-4. 
12. Flechner S. Psicoanálisis y cultura: la clínica actual de pacientes adolescentes en riesgo: un nuevo desafío? Revista Latinoamericana de Psicoanálisis 2000; 4:467-82.

13. Beautrais AL. Child and young adolescent suicide in New Zealand. Aust N Z J Psychiatry 2001; 35: 647-53.

14. Botega NJ, Barros MBA, Oliveira HB, Dalgalarrondo P, Marín-León L. Suicidal behavior in the community: prevalence and factors associated with suicidal ideation. Rev Bras Psiquiatr 2005; 27: 45-53.

15. Haarasilta L, Marttunen M, Kaprio J, Aro H. Major depressive episode and physical health in adolescents and young adults: results from a populationbased interview survey. Eur J Public Health 2005; 15:489-93.

16. World Health Organization. Child and adolescent health and development. http://www.who.int/ chp/gshs/methodology/en/index.html (acessado em Jul/2005).

17. Associação Brasileira de Empresas de Pesquisa. Critério de classificação econômica Brasil. http:// www.abep.org/codigosguias/ABEP_CCEB.pdf (acessado em Mar/2005).

18. Cordás TA, Castilho S. Imagem corporal nos transtornos alimentares. Instrumento de avaliação: Body Shape Questionnaire. Psiquiat Biol 1994; 2:17-21.

19. Cooper PJ, Taylor MJ, Cooper Z, Fairbum CG. The development and validation of the body shape questionnaire. Int J Eat Disord 1987; 6:485-94.

20. Barros A, Hirakata V. Alternatives for logistic regression in cross-sectional studies: an empirical comparison of models that directly estimate the prevalence ratio. BMC Med Res Methodol 2003; 3:21.

21. Hawton K, Rodham K, Evans E, Weatherall R. Deliberate self harm in adolescents: self report survey in schools in England. BMJ 2002; 325:1207-11.

22. Marcondes Filho W, Mezzaroba L, Turini CA, Koike A, Motomatsu Jr. A, Shibayama EEM, et al. Tentativas de suicídio por substâncias químicas na adolescência e juventude. Adolesc Latinoam 2002; 3:0-0.

23. Marín-León L, Barros MBA. Mortes por suicídio: diferenças de gênero e nível socioeconômico. Rev Saúde Pública 2003; 37:357-63.

24. Pinho MD, Berquó E, Oliveira KA, Lopes F, Lima LCA, Pereira N. Juventude, raça e vulnerabilidade. Rev Bras Estud Popul 2002; 19:277-94.

25. Botega NJ, Werlang BSG. Avaliação e manejo do paciente. In: Werlang BSG, Botega NJ, organizadores. Comportamento suicida. Porto Alegre: Editora ArtMed; 2004. p. 123-40.

26. Organização Mundial da Saúde. Prevenção do Suicídio: um manual para clínicos gerais. http://www. who.int/mental_health/prevention/suicide/en/ suicideprev_gp_port.pdf (acessado em Nov/2007).
27. Piccinelli M, Wilkinson G. Gender differences in depression: critical review. Br J Psychiatry 2000; 177:486-92.

28. Baptista MN, Assumpção Jr. FB. Epidemiologia e gênero na depressão. In: Baptista MN, Assumpção Jr. FB, organizadores. Depressão na Adolescência: uma Visão Multifatorial. São Paulo: E.P.U.; 1999. p. 57-68.

29. Wermeiren R, Ruchkin V, Leckman P, Deboutte D, Schwab-Stone M. Exposure to violence and suicide risk in adolescents/; a community study. J Abnormal Child Psychol 2002; 30:529-37.

30. Organização Mundial da Saúde. Departamento de Saúde Mental. Prevenção do suicídio: um manual para professores e educadores. http://www.who. int/mental_health/prevention/suicide/en/suici deprev_educ_port.pdf (acessado em Nov/2007).

31. Ruchkin VV, Stone MS, Koposov RA, Vermeiren R, King RA. Suicidal ideations and attempts in juvenile delinquents. J Child Psychol Psychiatry 2003; 44:1058-66.

32. Oquendo MA, Dragatsi D, Harkavy-Friedman J, Dervic K, Currier D, Burke AK, et al. Protective factors against suicidal behavior in Latinos. J Nerv Ment Dis 2005; 193:438-43.

33. Gould M, Velting D, Kleinman M, Lucas C, Thomas JG, Chung M. Teenagers' attitudes about coping strategies and help-seeking behavior for suicidality. J Am Acad Child Adolesc Psychiatry 2004; 43:1124-33.

34. Joiner TE, Pfaff JJ, Acres JG. Characteristics of suicidal adolescents and young adults presenting to primary care with non-suicidal (indeed non-psychological) complaints. Eur J Public Health 2002; 12:177-9.

35. Christoffersen MN, Poulsen HD, Nielsen A. Attempted suicide among young people: risk factors en a prospective register based study of Danish children born in 1966. Acta Psychiatr Scand 2003; 108:350-8.

36. Wild LG, Flisher AJ, Bhana A, Lombard C. Associations among adolescent risk behaviours and selfesteem in six domains. J Child Psychol Psychiatry 2004; 45:1454-67.

37. Palazzo LS, Béria JU, Alonso-Fernández F, Tomasi E. Depresión en la adolescencia en centros de atención primaria: importancia de un problema oculto en salud colectiva. Aten Primaria 2001; 28:543-9.

Recebido em 23/Jan/2008

Versão final reapresentada em 28/Abr/2008

Aprovado em 26/Mai/2008 